\title{
Impact of age, nutrition and metabolic factors on methylation status of CpG islands in Wnt-related genes of the human colon
}

\author{
N. J. Belshaw ${ }^{1}$, H. Tapp ${ }^{1}$, D. M. Bradburn ${ }^{3}$, D. M. Commane ${ }^{2}$, R. Arasaradnam ${ }^{2}$, J. C. Mathers ${ }^{2}$ \\ and I. T. Johnson ${ }^{1}$ \\ ${ }^{1}$ Institute of Food Research, Norwich Research Park, NORWICH, NR4 7UA, UK, ${ }^{2}$ Newcastle University, Human Nutrition \\ Research Centre, Institute for Ageing and Health, Framlington Place, NEWCASTLE-ON-TYNE, NE24HH, UK and \\ ${ }^{3}$ Wansbeck Hospital, Woodhorn Lane Ashington, Northumberland, UK
}

\begin{abstract}
Aberrant DNA methylation occurs during the development of tumours in the human colon, and probably contributes to the dysregulation of signalling pathways associated with cell proliferation, differentiation and apoptosis. CpG island (CGI) methylation also occurs progressively with age in some genes in epithelial cells within the apparently normal crypt; this may eventually increase the vulnerability of the mucosal field to neoplastic transformation. Although the effects of age are well established, it is not yet clear as to whether the onset of CGI methylation is also influenced by diet, metabolic status or environmental factors over the lifetime of an individual. In this crosssectional study, we used a highly sensitive, quantitative DNA assay to investigate the methylation status of genes associated with the Wntsignalling pathway in colonic biopsies from patients undergoing diagnostic colonoscopy, but shown to be free of disease.

Biopsies were obtained from 212 volunteers (95 males, 117 females; median age 50 years, range 17-78 years), together with blood samples and anthropometric data. Each volunteer also completed questionnaires for the assessment of dietary intake and physical activity. Ethical approval was received from the Northumberland Local Research Ethics Committee. DNA was extracted and subjected to bisulphite conversion, followed by quantification of CGI methylation, as previously described ${ }^{(1)}$. A total of eight Wnt-related genes (HPPl, $A P C$, SFRP1, SFRP2, DKK1, SOX17, AXIN2 and WIF-1) were assayed, together with LINE-1, as an index of global DNA methylation. The dependency of CGI methylation on selected co-factors (age, sex, height, weight, BMI, fatness index, waist:hip ratio, red cell folate, plasma folate, plasma homocysteine, plasma selenium, white cells and monocytes) was assessed for 190 study participants by stepwise linear regression. For each gene, candidate models were generated by re-sampling of 10000 datasets, using a $P$-value of 0.05 as the criterion for the inclusion of each variate in the model. To minimise over-fitting, the performance of candidate models was compared by 500 rounds of cross-validation, using a 65:35 split for the training:test segments, and the models with the lowest standard error in prediction (SEP) were selected.

Average CGI methylation for Wnt-related genes varied markedly, from $0.11 \%$ for $D K K 1$ to $6.86 \%$ for WIF1. For three genes $(D K K 1$, $A X I N 2$ and $A P C$ ) CGI methylation showed no statistically significant dependency on any co-factor. For WIF1, HPP1, SOX17 and SFRP2, CGI methylation increased significantly with age $\left(P<0.1 \times 10^{-5}\right)$; SFRP1 showed a less significant positive correlation $\left(P<0.1 \times 10^{-2}\right)$, whereas LINE1 methylation declined with age $(P<0.05)$. For WIF1, SFRP1 and HPP1, CGI methylation was positively, though weakly, correlated with serum folate $(P<0.05)$, and for WIF1 there was a weak negative correlation with serum selenium concentration $(P<0.05)$. There was some evidence for a positive correlation between CGI methylation and BMI for WIFl only $(P<0.05)$. We conclude that the Wnt-related genes of colorectal epithelial cells differ markedly in their susceptibility to age-related CGI methylation, but for highly methylated genes, other factors, including folate and selenium status, may influence the changes in CGI methylation across the lifespan.
\end{abstract}

We thank the Food Standards Agency (N12015) and the BBSRC for financial support.

1. Belshaw NJ, Elliott GO, Foxall RJ et al. (2008) Br J Cancer 8, 136-142. 